\title{
Per una nuova assiologia del consumo Una proposta di ampliamento, approfondimento e aggiornamento del modello flochiano
}

\author{
Antonio Laurino \\ Università di Bologna - Alma Mater Studiorum; Università della Repubblica di San Marino \\ antonio.laurino@outlook.com
}

\begin{abstract}
This essay illustrates the genesis, potential and limits of the square of consumption values, conducting both a theoretical overview and an empirical investigation, focused on the Italian context. The treatment takes into account the main readings and applications proposed by scholars and professionals as much as the analysis of a large textual corpus that aims to represent the contemporary advertising landscape. As a result, it is possible to make a proposal aimed at integrating, deepening and updating the original model, in accordance with the flochian principle whereby the semiotic perspective can bring several benefits to communication in terms of greater intelligibility, relevance and differentiation.
\end{abstract}

\section{Keywords}

Semiotics of consumption, Semiotics of advertising, Jean-Marie Floch, Semiotic square, Values

\section{Sommario}

Introduzione

1. Genesi e fortuna di un modello

2. La ricognizione teorica

3. Per un ritorno alla realtà testuale

4. L'indagine empirica

Conclusione

Bibliografia 


\section{Introduzione}

Il quadrato dell'assiologia del consumo è una pietra miliare della semiotica della pubblicità. Elaborato nel 1990 dal semiologo e consulente francese JeanMarie Floch, ha ben presto superato i confini della semiotica applicata ed è entrato a far parte degli strumenti operativi a disposizione dei ricercatori di marketing. Negli anni è stato oggetto di numerose riprese e rielaborazioni che ne hanno evidenziato limiti e potenzialità, ma la sua applicazione è tuttora diffusa e apprezzata. Eppure, ci si potrebbe ragionevolmente chiedere se i cambiamenti intercorsi nel panorama pubblicitario dal momento della sua elaborazione non ne abbiano in qualche modo intaccato l'attualità e, dunque, l'utilità. Si tenterà di rispondere a questa domanda, operando sia una ricognizione teorica sia un'indagine empirica. Si partirà dall'introdurre il modello, focalizzandosi sulle caratteristiche alla base della sua fortuna; si cercherà di fare il punto sulle riletture di cui è stato oggetto da parte di studiosi e professionisti; si illustrerà nel dettaglio il processo che ha portato alla definizione di un corpus sufficientemente ampio e vario da poter aspirare a essere rappresentativo della realtà pubblicitaria contemporanea; si procederà all'analisi dei testi e, sulla base delle evidenze empiriche, si avanzeranno alcune proposte volte ad ampliare, approfondire e aggiornare le categorie flochiane originarie.

\section{Genesi e fortuna di un modello}

A partire dall'analisi di un grande numero di annunci stampa e spot del settore automotive, Floch (1990) elabora una tipologia dei possibili modi in cui può essere valorizzato l'oggetto automobile. Per farlo utilizza e mette alla prova alcuni strumenti teorici di grammatica narrativa. In particolare, riprende la distinzione tra valori di base e valori d'uso: i primi «corrispondono al piano delle preoccupazioni fondamentali dell'essere e di cui la ricerca sottende la vita e conferisce senso alla realizzazione di molteplici programmi d'azione secondari, più superficiali, "più pratici”» (Floch 1990: 172 tr. it.) espressione, appunto, di valori d'uso.

Floch osserva come nei testi pubblicitari la relazione che lega valori di base e valori d'uso non sia di implicazione, ma di contrapposizione. In altre parole, mentre in una narrazione si è soliti ragionare in termini di presupposizione tra azioni volte al raggiungimento di un obiettivo finale e azioni legate all'acquisizione di oggetti o capacità strumentali, in pubblicità si assiste spesso a un'opposizione paritetica tra fini e mezzi: «è come se si dovesse - a priori scegliere tra l'utilizzazione di una vettura che risponde ai vostri bisogni e il possesso, addirittura la gioia, di una vettura che corrisponde ai vostri gusti o ai vostri desideri» (Ibidem).

Constatato ciò, Floch riconduce ai valori d'uso un tipo di valorizzazione che definisce pratica, e a quelli di base un tipo che definisce utopica. In seguito, articolando questa opposizione semantica fondamentale sul quadrato 
Antonio Laurino | Per una nuova assiologia del consumo

semiotico, ${ }^{1}$ individua due ulteriori forme di valorizzazione che chiama critica e ludica, ${ }^{2}$ corrispondenti rispettivamente alla negazione dei valori di base e alla negazione dei valori d'uso. Lo schema che ne risulta è il seguente:

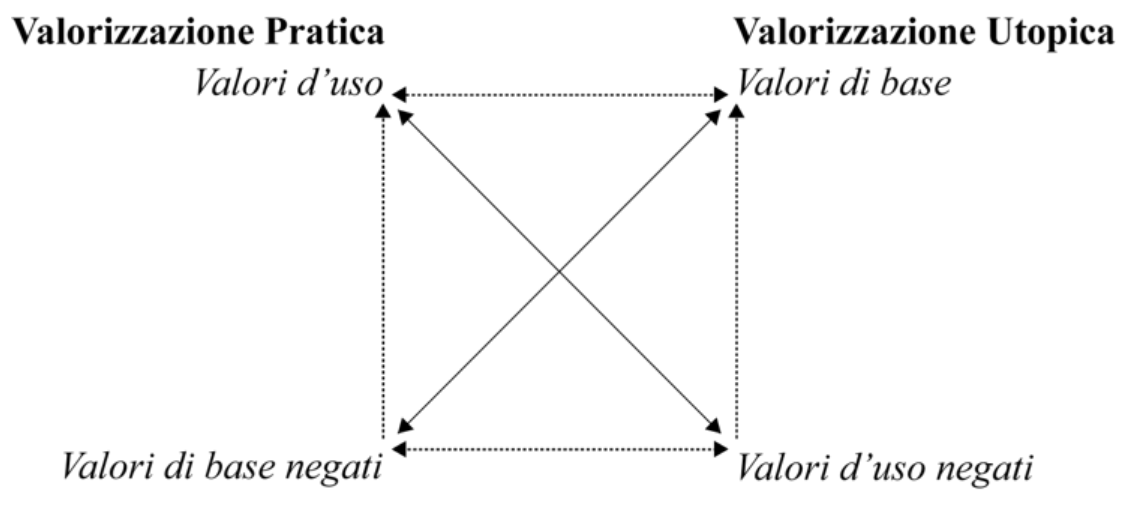

Valorizzazione Critica

Valorizzazione Ludica

Figura 1. Quadrato dell'assiologia del consumo elaborato da Floch (1990).

${ }^{1}$ Il quadrato semiotico è il modello elaborato da Algirdas J. Greimas (Greimas e Courtés 1979) attraverso il quale è possibile visualizzare le relazioni logiche che regolano un micro-universo di senso, come un singolo testo o un insieme di testi. Il processo di costruzione di un quadrato parte dall'individuazione di una categoria semantica formata da due termini in relazione di contrarietà $(a V s b)$. Tale categoria viene espansa attraverso l'individuazione dei rispettivi contraddittori (non- $b$ e non- $a$ ), ottenuti negando i termini di partenza. Essi, a loro volta, si trovano in relazione di contrarietà. Inoltre, in un quadrato ben posto, ciascuno di questi due termini intrattiene una relazione di implicazione con il termine opposto rispetto a quello da cui è stato generato per negazione. Graficamente:

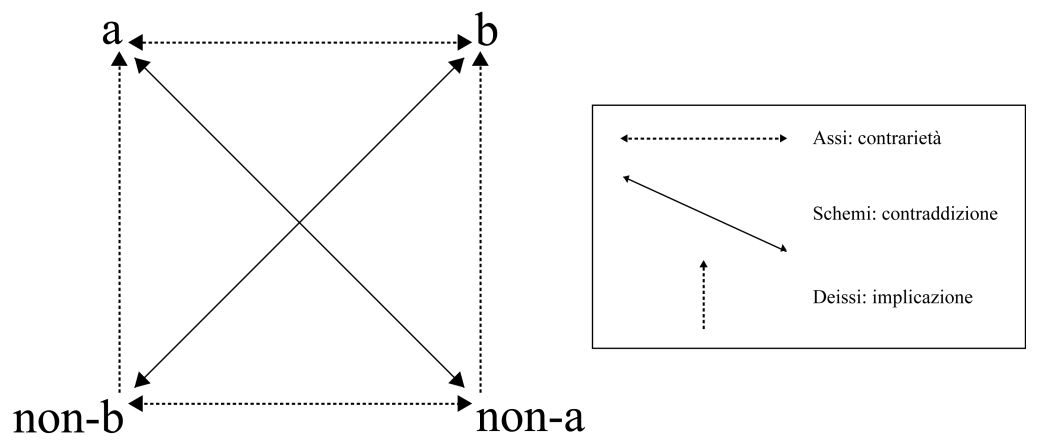

${ }^{2}$ Nonostante Floch tenti di lessicalizzare le quattro forme di valorizzazione nel modo più trasparente possibile, le etichette scelte restano arbitrarie e in qualche modo opache. Vale la pena di precisare, dunque, che nel caso dei valori di base il termine "utopico" "non è qui da prendere in senso di illusorio, ma proprio nel senso di tensione finale (ideale)» (Floch 1990: 175 tr. it.); nel caso del "ludico" ci si riferisce a un'attività scevra da qualsiasi motivazione o ripercussione pratica; infine, il termine "critico" indica «una logica dell'esame, del distanziamento e del calcolo» (ivi: 176). 
Le quattro forme di valorizzazione individuate sono:

- la valorizzazione pratica (corrispondente ai valori d'uso) che si basa sulla magnificazione delle caratteristiche funzionali dell'oggetto e alla quale, nel settore merceologico considerato, possono essere ricondotte le caratteristiche di maneggevolezza, comfort o robustezza dell'auto;

- la valorizzazione utopica (corrispondente ai valori di base) che chiama in causa valori esistenziali, ossia legati a un vero e proprio progetto di vita, come lo spirito di avventura o la ricerca della libertà;

- la valorizzazione ludica (corrispondente alla negazione dei valori d'uso) che esalta il prodotto in quanto fonte di piacere, svago e divertimento, e che si concretizza nelle caratteristiche di lusso, gratuità o raffinatezza dell'oggetto;

- la valorizzazione critica (corrispondente alla negazione dei valori di base) che prevede una sorta di distanza cognitiva nella valorizzazione dell'oggetto e si traduce nella creazione di rapporti qualità/prezzo o costi/benefici.

Nel loro complesso, questi quattro modi di valorizzare l'oggetto pubblicitario formano un «sistema dei valori di consumo» (Floch 1990: 163 tr. it.), ossia un'assiologia.

Nonostante sia stato elaborato a partire da un settore specifico, il quadrato dell'assiologia del consumo si è rivelato uno strumento utile a rendere conto dei posizionamenti sia di prodotti afferenti a settori merceologici anche molto distanti dal segmento automotive, sia delle marche (cfr. Floch 2013; Marrone 1999, 2007; Ceriani 2001). I quattro termini del quadrato, infatti, vanno intesi come «serbatoi semantici» (Zannin 2009: 104), forme vuote suscettibili di essere riempite di valori specifici a seconda del prodotto, della congiuntura e, in ultima analisi, del singolo testo pubblicitario.

Non è difficile immaginare, ad esempio, uno spot di un detersivo in cui il prodotto viene magnificato per il suo potere pulente (valorizzazione pratica); di un amaro che diventa espressione di uno stile di vita avventuroso (valorizzazione utopica); di un pc portatile di cui si sottolinea l'impareggiabile rapporto tra prestazioni e volume occupato (valorizzazione critica); di uno smartphone per il quale a essere messo in luce è il piacere/divertimento che il suo utilizzo procura (valorizzazione ludica).

Si tratta dunque di un modello che fin dalla sua introduzione ha presentato notevoli potenzialità di generalizzazione; non a caso, già nelle pagine conclusive del suo saggio, Floch intravedeva la possibilità che potesse essere alla base di «una assiologia della vita quotidiana e non del solo consumo» (Floch 1990: 190 tr. it.). E, in effetti, si potrebbe sostenere la validità per così dire universale di questi quattro termini: pratico, utopico, critico e ludico rappresenterebbero cioè quattro modi di guardare all'oggetto pubblicizzato (e al mondo) necessari e sufficienti. Allo stesso tempo, però, tale ipotesi ha spesso portato ad applicazioni meccaniche e banalizzanti del modello flochiano, che rischiano di giungere a una tanto sostanziale, quanto inutile, indifferenziazione. Occorre dunque volgere lo sguardo alla letteratura di 
Antonio Laurino | Per una nuova assiologia del consumo

settore e andare alla ricerca di letture e integrazioni in grado di accrescere ulteriormente la potenza operativa di questo strumento.

\section{La ricognizione teorica}

Il portato euristico del modello flochiano è stato compreso e discusso da numerosi autori, non solo semiotici, che ne hanno proposto riletture e applicazioni, dal momento che è unanimemente riconosciuto

quanto stia nei compiti primari dell'azione pubblicitaria quello di valorizzare un prodotto, un servizio o un marchio. Né i prodotti (o i servizi, o i marchi) da un lato, né i valori dall'altro lato, vengono costruiti in sede di azione pubblicitaria; ciò che in tale sede viene compiuto è solo un atto di connessione tra entità appartenenti a due differenti “universi": l'universo dei prodotti da un lato e quello dei significati, dei valori, delle idee culturalmente rilevanti dall'altro lato. (Ferraro 1999: 26)

Lo stesso Floch (1995), alcuni anni dopo la sua elaborazione, intervenne nuovamente sul modello rinominando la valorizzazione ludica in ludicoestetica e prevedendo così l'eventuale magnificazione dell'oggetto per le sue caratteristiche estetiche e/o per il suo design. Se da un lato un'operazione del genere rimediava a una lacuna piuttosto evidente del modello originario, dall'altro ne intaccava la purezza formale, dal momento che le posizioni articolate sul quadrato sono diventate cinque. Come nota Maria Pia Pozzato (2001), infatti, ludico ed estetico sono accomunate dall'essere entrambe espressione della negazione dei valori d'uso (e quindi della valorizzazione pratica) ma mantengono comunque delle notevoli differenze che non solo rendono la loro giustapposizione piuttosto problematica, ma non escludono che in uno stesso testo possano trovarsi persino contrapposte.

Gianfranco Marrone (2001) mette in luce un ulteriore aspetto problematico del quadrato flochiano. Quest'ultimo, infatti, «sovrappone e finisce per schiacciare l'uno sull'altro due diversi livelli di pertinenza della struttura narrativa: quello dei valori profondi [...] e quello dei programmi narrativi» (Marrone 2001: 187). Così, all'interno del polo ludico, troviamo spot nei quali si vede il soggetto divertirsi utilizzando l'oggetto, ma anche annunci in cui al centro della narrazione vi è l'oggetto valorizzato per le sue qualità estetiche. All'interno di ogni categoria, dunque, sarebbe possibile rinvenire tre diverse prospettive narrative: una legata al soggetto, una all'oggetto e una incentrata sulla relazione tra i due. Schematizzando: 


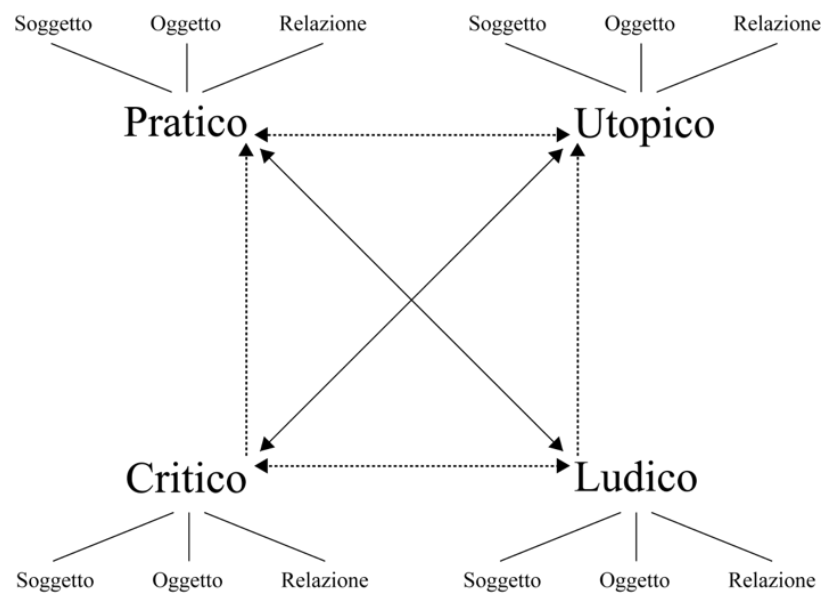

Figura 2. Rielaborazione dell'assiologia del consumo ad opera di Marrone (2001).

Sul piano operativo questa integrazione ha il merito di rendere conto in modo più completo della ricchezza narrativa dei testi pubblicitari, affinando ulteriormente le potenzialità analitiche del modello.

Di tutt'altro genere sono le osservazioni di Ugo Volli (2003), secondo il quale i vertici superiori del quadrato (pratico e utopico) riguarderebbero valori che si possono considerare inerenti all'oggetto in sé, mentre quelli inferiori (critico e ludico) coinvolgerebbero dimensioni attribuite all'oggetto dalla comunicazione pubblicitaria. Inoltre, i vertici di destra articolerebbero qualità immateriali, intangibili, mentre quelli di sinistra qualità essenzialmente concrete e tangibili. Un'interpretazione di questo tipo, però,

invece di insistere su posizioni formali e narrative (rapporto del soggetto con l'oggetto) sembra ritornare a spiegazioni in qualche modo referenzialiste che distinguono in base alla sostanza stessa dei valori: materiali vs immateriali, funzionali vs emozionali sull'asse orizzontale; valori dell'oggetto in sé, intrinseci, vs valori apportati dalla comunicazione pubblicitaria, costruiti da discorsi secondi, sull'asse verticale. (Zannin 2009: 107)

Inoltre, nulla vieta di considerare i valori estetici (e per certi versi anche quelli critici) come inerenti all'oggetto in sé e non apportati esclusivamente dalla comunicazione.

Cinzia Bianchi (2005), dal canto suo, pone l'accento su un punto di forza del modello flochiano: il quadrato semiotico, infatti, oltre a permettere un'organizzazione paradigmatica, ossia statica, dei vari concetti, consente anche una loro lettura sintagmatica, cioè dinamica, in grado di rendere conto delle evoluzioni di posizionamento di un singolo prodotto/servizio o di una marca nel tempo. Attraverso le operazioni logiche di negazione (passaggio dal termine $a$ al suo contraddittorio non- $a$ ) e di affermazione (passaggio da non$a$ al suo complementare $b$ ) è possibile tracciare sul quadrato un percorso ad "ali di farfalla" e quindi, entro certi limiti, prevedere verso quale polo potrebbe (e dovrebbe) tendere un prodotto o una marca che va alla ricerca di un 
Antonio Laurino | Per una nuova assiologia del consumo

posizionamento maggiormente distintivo rispetto ai concorrenti. Al di là di questo, però, nella sua rilettura di Floch, Bianchi si sofferma sulla possibilità di tenere traccia delle trasformazioni valoriali all'interno di un singolo testo pubblicitario, in particolar modo di uno spot. Se per un verso questa lettura rischia di apparire troppo stringente, dall'altro chiama in causa una situazione facilmente riscontrabile in fase di analisi: la compresenza di più forme di valorizzazione all'interno di un unico annuncio o spot. Vale la pena di notare che in tutti questi casi, vista la natura esclusiva e, da questo punto di vista, eccessivamente rigida del quadrato semiotico, si è soliti ragionare in termini di valorizzazioni dominanti, polarizzando il testo attorno a un singolo vertice del quadrato.

Una possibile soluzione a questo limite viene fornita da Andrea Semprini (1992), il quale riprende i quattro termini del quadrato flochiano (pratico, utopico, critico, ludico), ma li dispone in modo da formare un mapping, «ovvero un sistema logico che oppone, incrociandole, due categorie differenti» (Semprini 1992: $121 \mathrm{tr}$. it.): sull'asse verticale trovano posto i due termini contrari pratico/utopico, mentre su quello orizzontale i due subcontrari critico/ludico che vanno così a specificare le aree semantiche definite dai primi due. In questo modo l'autore punta a produrre una rappresentazione concettuale maggiormente familiare ai professionisti del marketing; rappresentazione che, a differenza del quadrato semiotico, consenta di cogliere sovrapposizioni e sfumature di senso mescolando a due a due le categorie originarie. Inoltre, l'incrocio delle quattro dimensioni dà vita a dei quadranti (denominati missione, informazione, euforia e progetto) all'interno dei quali è possibile procedere al posizionamento di prodotti e marche.

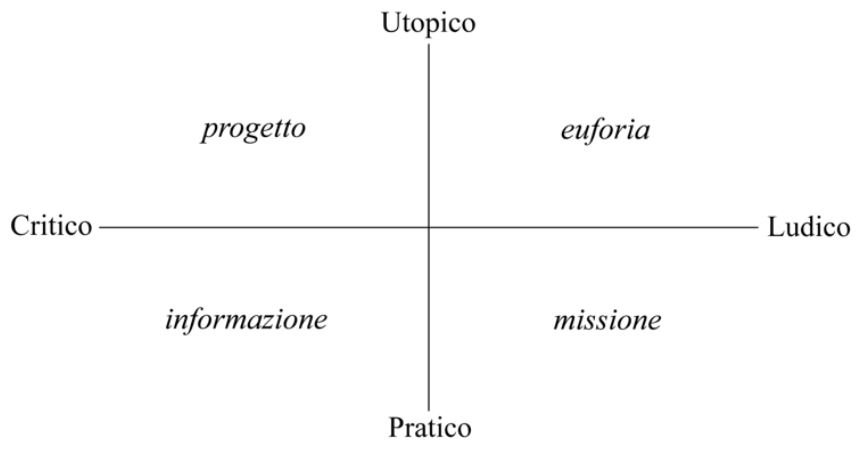

Figura 3. Mapping semiotico dei valori del consumo proposto da Semprini (1992).

Pur possedendo diversi vantaggi operativi, sul piano teorico la proposta di Semprini è stata oggetto di svariate critiche, tra cui quelle sollevate da Giulia Ceriani (2001), la quale contesta a questa schematizzazione:

il fatto di voler discendere da un quadrato semiotico e, nella fattispecie, di utilizzare la terminologia propriamente legata a un quadrato ben definito, appunto quello dell'assiologia dei valori di consumo. In quest'ultimo, i due poli subcontrari, critico e 
ludico, vengono definiti per negazione rispetto ai contrari rispettivi, utopico e pratico. Se non si ottengono per negazione - ma sono indipendenti e costituiscono un'altra categoria - questi due termini assumono un senso diverso, per cui sarebbe corretto utilizzare un'altra terminologia capace di enunciare chiaramente la differenza tra i due utilizzi.

In secondo luogo, non si vede come questi due subcontrari possano rappresentare le qualificazioni e le specificazioni di termini di cui essi sono precisamente la negazione e la contraddizione.

Infine, il terzo problema, e probabilmente il più grave, è quello dell'articolazione di una schematizzazione "assoluta" e non dipendente da un contesto dato. Ora, non si può mai ottenere un quadrato in assoluto, dato che la categoria che esso apre è sempre derivata da un testo analizzato di cui si è trovata l'isotopia fondamentale. Così, i quattro termini relativi ai valori - già generici nel quadrato di Floch ma per lo meno interdefiniti - sono qui talmente generici da diventare purtroppo inutili. (Ceriani 2001: 115-116)

Per questi motivi, il mapping dei valori di consumo, pur ispirandosi apertamente all'assiologia flochiana, sembra mantenerne in realtà le sole etichette terminologiche, presentandosi come qualcosa di profondamente diverso da essa. D'altro canto, la proposta di Semprini fa emergere la discussa questione dell'abbandono o meno del modello del quadrato semiotico: da un lato, trattandosi di un quadrato a priori, c'è da chiedersi quanto riesca a rendere conto esaustivamente di una realtà complessa e cangiante come quella pubblicitaria; dall'altro, la sua tenuta logica viene messa in discussione, dato che le quattro valorizzazioni rappresenterebbero aree semantiche troppo ampie per poter essere in relazione di contrarietà, contraddizione e implicazione.

\section{Per un ritorno alla realtà testuale}

Le osservazioni e le riflessioni fin qui riportate si devono in gran parte a studiosi che si dedicano anche ad attività di consulenza o ricerche di mercato; autori che si servono della semiotica per "mordere sul reale" - usando un'espressione dello stesso Floch - e che si scontrano con la difficoltà di riuscire a rendere conto di un panorama competitivo e di una società sempre più complessi e frammentati. Alla luce di ciò, è lecito domandarsi se i grandi mutamenti di scenario intercorsi dall'epoca dell'introduzione dello strumento flochiano non ne abbiano in qualche modo intaccato l'attualità operativa. Si tratta di profonde trasformazioni che hanno riguardato tanto il sistema economico e pubblicitario quanto il tessuto socioculturale nel suo complesso.

Intorno alla metà degli anni Ottanta - periodo in cui Floch mette a punto la sua proposta - l'industria pubblicitaria godeva di un momento di particolare dinamismo, frutto anche di una fase di espansione del sistema economico mondiale. In occidente si assisteva a una crescita costante degli investimenti pubblicitari, trainati dal medium televisivo, e in crescita erano anche il benessere e il piacere del consumo di fasce sempre più ampie della popolazione. Si era, insomma, in Francia come in Italia, in quella che viene generalmente considerata l'epoca d'oro della pubblicità. Un'epoca, in cui, tra 
Antonio Laurino | Per una nuova assiologia del consumo

l'altro, le si riconosceva una notevole importanza da un punto di vista sia economico sia sociale. Da allora molte cose sono cambiate, non soltanto in termini di nuovi assetti e coordinate socioculturali di ordine generale, ma anche, e forse soprattutto, per ciò che riguarda il rapporto tra individuo, comunicazione e consumo. Gli elementi di discontinuità sono numerosi, e tra questi vanno menzionati:

- le radicali innovazioni che l'ascesa delle tecnologie e delle reti digitali ha introdotto nei modelli di fruizione dei contenuti e nelle forme di interazione tra i vari soggetti coinvolti nel flusso della comunicazione e della stessa produzione: un rapporto sempre più paritetico e improntato alla co-creazione;

- il fiorire di approcci e teorie che hanno posto l'accento sul consumatore e su alcuni dei suoi tratti fino ad allora poco indagati: la sua dimensione emotiva e passionale; le implicazioni relazionali dei suoi comportamenti; la centralità della dimensione estesica ed estetica nelle sue pratiche di consumo; le sue esperienze di acquisto e fruizione dei prodotti/servizi (cfr. Pine e Gilmore 1999; Ferraresi e Schmitt 2006);

- le cicliche ondate di protesta nei confronti dei grandi brand e della stessa pubblicità (cfr. Klein 2000), che hanno contribuito a creare una nuova, diffusa sensibilità nei confronti dell'universo del consumo, delle sue dinamiche e delle sue implicazioni.

Tutto ciò nel quadro di un progressivo calo degli investimenti pubblicitari che dagli inizi del nuovo millennio giunge fino ai giorni nostri.

Questi fattori hanno contribuito a forgiare il cosiddetto consumatore postmoderno:

un soggetto disincantato e laico, autonomo e responsabile, consapevole che i consumi sono ormai anche una forma di linguaggio con cui comunica, a se stesso e agli altri, brani della propria identità, i propri stati d'animo o i mood del momento; che ha un rapporto sovente ludico e privo di soggezione con le merci; capace però di nuovi incantamenti che non hanno niente a che spartire con il feticismo delle merci; che si avvale con interesse - sia pure per utilizzi molto diversi dai significati attribuiti dalla produzione e dalle istruzioni per l'uso - delle proposte del mercato. (Fabris 2009: 143)

Un consumatore che, in quanto target, ha messo in crisi tutti i tradizionali sistemi di categorizzazione, ponendo sfide sempre nuove a creativi e agenzie pubblicitarie, che hanno il compito di colpire nel segno.

Detto ciò, viene da chiedersi in che modo e fino a che punto tutti questi aspetti socio-economico-culturali possano influenzare la produzione pubblicitaria. A tal proposito, occorre osservare che

i messaggi pubblicitari vengono realizzati sulla base dei linguaggi, dei valori, delle forme narrative, delle competenze musicali eccetera dei propri destinatari. Da questo punto di vista [...] ben lungi dal presentarsi come attività propriamente creativa, il lavoro dell'agenzia pubblicitaria ci appare piuttosto come un abile lavoro di confezionamento, di rielaborazione e riadattamento di elementi e configurazioni affermate sulla scena culturale. (Ferraro 1999: 56-57) 
In altri termini, per quanto si sia spesso evidenziato il potere della pubblicità di proporre (e influenzare) non solo scelte di consumo, ma anche stili di vita e sistemi di valori, quello che qui interessa mettere in luce è ciò che sta a monte, ossia l'opera di selezione di alcuni tratti della realtà sociale che il pubblicitario mette in atto al momento della progettazione di una campagna: valori, gusti, estetiche vengono accuratamente scelti per essere il più possibile in linea con quelli di un determinato target. A questo punto, il lavoro del creativo sta nell'ancorare nel modo più efficace il prodotto, il servizio o il brand ai caratteri selezionati; che ciò avvenga attraverso una ricerca sistematica o che si basi invece sull'intuizione, quel che conta è che la pubblicità nella sua produzione e ri-produzione cristallizza determinati tratti di una società, di un'epoca e di una cultura per proporli a quei soggetti che, in modo più o meno consapevole, li hanno precedentemente messi in scena.

Dunque, è lecito ipotizzare che l'insorgere e l'affermarsi di nuove tendenze, sensibilità ed estetiche nel tessuto sociale abbia influito sulla produzione pubblicitaria, in particolare per ciò che riguarda le forme di valorizzazione. Da qui l'interesse e l'opportunità di verificare l'attualità operativa del modello flochiano. Per farlo si volgerà lo sguardo alla realtà testuale, prendendo in considerazione prodotti comunicativi concreti, al fine di ri-mettere alla prova dei testi quanto di fatto ha avuto fin da subito una forte ambizione di generalità.

\subsection{Il corpus: rischi e linee guida nella sua costruzione}

In che modo è possibile procedere a un'operazione di questo tipo? Quali e quanti testi dovranno essere presi in considerazione, di quale settore merceologico e all'interno di quale intervallo temporale? Insomma, a emergere con forza è la delicata ma cruciale questione della costruzione del corpus. Se gli strumenti semiotici hanno dimostrato spesso di operare egregiamente su oggetti "concreti” e ben definiti (spot, affissioni, annunci stampa, ecc.) e se le osservazioni che ne derivano possono aspirare a un certo grado di generalizzazione, ciò è dovuto, ancor prima che alle capacità del ricercatore, alla perizia con la quale egli individua il suo orizzonte di azione. Non una qualsiasi collezione di testi può essere considerata un corpus, e la sua definizione è un'operazione che nasconde numerose insidie, in grado di condizionare l'esito dell'analisi. I principali rischi sono la scarsa rappresentatività, la scarsa osservabilità e il costruttivismo. Per scongiurarli, o quantomeno ridurne l'incidenza, occorre

- prendere in considerazione una quantità di oggetti che sia maneggiabile e adeguata alle risorse scarse dell'analista, ma che allo stesso tempo risulti rappresentativa dell'universo di cui si vuole rendere conto;

- che i testi selezionati sulla carta siano poi in concreto effettivamente reperibili per essere raccolti e osservati dall'analista;

- fare in modo che le ipotesi di partenza del ricercatore non lo influenzino a tal punto da mettere insieme un corpus volto essenzialmente a ritrovare ciò di cui è in cerca. 
Antonio Laurino | Per una nuova assiologia del consumo

Un buon corpus, dunque, dovrebbe essere significativo in termini di quantità e qualità dei testi, anche se non sarà (e non potrà mai essere) completo. Allo stesso tempo è basilare - ma non banale - che sia ben delimitato e non aperto. Non ultimo, dovrà essere adeguato a quello che è l'oggetto d'analisi. Vista la centralità di tale operazione, e tenendo a mente questi principi, ci si soffermerà ora sulla costruzione del corpus e si esporranno nel dettaglio le scelte che hanno portato alla sua definizione.

\subsection{Il corpus: verso la definizione}

L'universo della comunicazione commerciale è uno degli ambiti discorsivi più prolifici e cangianti della nostra società. L'altissima densità dei messaggi fa il paio con la loro rapida obsolescenza, a cui corrisponde una notevole velocità del processo di produzione. Isolare, in questo flusso incessante, un insieme definito, coerente e in qualche modo coeso non è cosa semplice. $\grave{E}$ necessario operare fin da subito delle scelte per certi versi arbitrarie ma il più possibile argomentate e coerenti con l'obiettivo della ricerca. Il primo passo, se si vuole immediato - ma per nulla scontato, vista la trasversalità dell'applicazione del modello - è stato scegliere un singolo settore merceologico sul quale concentrarsi. Per una serie di ragioni si è scelto il settore automotive.

In primo luogo, motivazioni di tipo filologico. Come si è detto, infatti, la tipologia flochiana delle forme di valorizzazione nasce proprio dall'analisi di campagne stampa e tv del settore automobilistico, realizzate in Francia tra l'inizio e la metà degli anni Ottanta. Fermo restando il differente contesto nazionale, 3 dunque, è sembrato particolarmente pertinente tornare a osservare il medesimo settore per poterne eventualmente rilevare differenze, evoluzioni e tendenze.

In secondo luogo, ragioni strategiche: i grandi gruppi automobilistici, infatti, sono stabilmente tra i maggiori investitori pubblicitari ed è lecito supporre che a un cospicuo e relativamente costante investimento pubblicitario da parte delle aziende corrisponda una notevole ricerca di concept distintivi e campagne innovative da parte di agenzie e creativi.

D'altra parte, pur restringendo il campo di indagine al solo settore automotive, restano aperte numerose questioni. La prima: su quale/i tipo/i di testo concentrare l'attenzione? Spot, annunci stampa, affissioni, banner, viral? O una combinazione di questi? Esigenze di economia e di osservabilità hanno reso necessario operare un'ulteriore selezione verso una forma specifica: lo spot; in virtù di considerazioni di ordine sia teorico sia pratico.

In primis, il formato audiovisivo pubblicitario consente, per sua stessa natura, maggiore ricchezza semantica ed espansione narrativa rispetto

3 Pur trattandosi di una differenza di non poco conto, si ritiene ragionevole riferirsi al solo contesto nazionale in virtù della larga diffusione e applicazione che le teorie flochiane hanno avuto (anche) in Italia. 
Antonio Laurino | Per una nuova assiologia del consumo

all'immagine fissa; il che potrebbe incidere sulle forme di valorizzazione a cui i pubblicitari fanno ricorso.

In secundis, alcune osservazioni empiriche sulle dinamiche di creazione e pianificazione delle campagne pubblicitarie indicano che laddove sono previste la realizzazione e la diffusione di un commercial, con una certa probabilità sarà presente anche una campagna stampa o di affissioni. Mentre, per evidenti ragioni di budget, lo stesso grado di correlazione non vale al contrario. Ciò chiama in causa sia il principio di coerenza sia quello di rappresentatività del corpus: la scelta di prendere in considerazione un unico tipo di testo, infatti, garantisce una maggiore uniformità in fase di analisi, ma può sollevare anche obiezioni in merito alla rilevanza, all'interno del panorama pubblicitario, della forma scelta. Ci si potrebbe chiedere, ad esempio, se sia corretto e opportuno tralasciare le forme innovative e maggiormente coinvolgenti legate alla rete. A tal proposito, Marco Lombardi (2007) osserva che, pur essendo nel pieno dell'era post spot, in cui le dinamiche e i modelli di interazione della rete sono ormai imprescindibili per il marketing, si continua ad aver bisogno di

una grande potente idea di comunicazione [...] e il modo più semplice per produrla è quella di avere uno spot tv al centro del piano multimedia off e on line. [...] Il suo ruolo [dello spot] sarà meno importante, alcune delle modalità media alternative alla tv generalista saranno le più rilevanti per ingaggiare la persona, ma la grande idea deve essere sintetizzata al centro per assicurare un insieme coeso. E la miglior sintesi, la più chiara e la più forte, rimane quella che scaturisce dalla ferrea disciplina del brevissimo tempo in video: il trenta secondi tv. (Lombardi 2007: 29-30)

Allo stesso tempo, se si intende lo spot come forma audiovisiva pubblicitaria, ci si rende immediatamente conto di quanto sia riduttivo ricondurlo al solo medium televisivo e al suo specifico tipo di fruizione: ci si imbatte regolarmente in video pubblicitari, tanto sul web - si pensi alle campagne (che aspirano a diventare) virali, incentrate proprio sulla realizzazione di prodotti audiovisivi, o alla pratica, diffusissima su portali di informazione e piattaforme con un alto numero di accessi, di inserire annunci pubblicitari prima dei propri contenuti - quanto in spazi outdoor, come aeroporti, stazioni ferroviarie e fermate della metropolitana.

Per questi motivi è sembrato possibile e proficuo concentrarsi su testi pubblicitari di natura audiovisiva.

D'altra parte, consapevoli della necessità di garantire un certo grado di varietà all'interno del corpus, non si è discriminato tra spot andati in onda su emittenti nazionali generaliste o tematiche, digitali terrestri o satellitari, in chiaro o a pagamento. Non sono state operate, inoltre, selezioni in base alle classificazioni di settore, al target $\mathrm{o}$ al posizionamento. Tutto ciò perché si può ipotizzare che in determinati segmenti vengano adottate più frequentemente alcune forme di valorizzazione. Dunque, sarebbe stato rischioso operare a priori differenziazioni di questo genere, pena una drastica riduzione della rappresentatività del corpus. 
Infine, è stato necessario definire un intervallo temporale abbastanza ampio da permettere la raccolta di un numero di elementi significativo ma gestibile. Si è così preso in considerazione un periodo di due anni, che va dal $1^{\circ}$ luglio 2011 al 30 giugno 2013.

\subsection{Il corpus: affinamento e chiusura}

Una volta scelti e debitamente argomentati i principi - pur sempre arbitrari - attraverso cui sono stati individuati gli elementi del corpus, restava da sciogliere il nodo della loro effettiva reperibilità e osservabilità. È questo un punto cruciale di qualsiasi indagine in estensione ed è anche quello che più spesso ne condiziona il buon esito. In questo caso, si è potuto usufruire di uno strumento che ha garantito un elevato livello di sistematicità nella raccolta degli spot: la piattaforma Creative Dynamix.4 Si tratta di un servizio web offerto dall'istituto Nielsen Media Research che consente di tenere traccia delle creatività diffuse sui principali mezzi e di visionarle online dopo il loro lancio.

Impostando i diversi parametri sulla base delle riflessioni fatte fin qui e affinando ulteriormente $\mathrm{i}$ risultati di ricerca iniziali, si è giunti finalmente alla definizione di un corpus ben delimitato e facilmente osservabile. In un primo momento, si è operato sulle variabili di ricerca che riguardavano:

- il settore merceologico (automobilistico);

- le diverse categorie di settore individuate dalla Sistematica AdEx (campagne istituzionali, fuoristrada, lusso, medio alto, medio inferiore, monovolume, multisegmenti, sport, superiore, superutilitarie, utilitarie, microcars);

- il tipo di creatività (spot);

- i canali di diffusione (tv);

- l'arco temporale (2 anni).

- In questo modo gli item restituiti dal sistema ammontavano a 1915. Nonostante il notevole grado di personalizzazione, però, ci si è resi conto che era necessario operare un'ulteriore scrematura. Ai fini della ricerca, infatti, sono apparsi poco pertinenti e quindi trascurabili:

- gli inviti all'ascolto e le citazioni;

- i diari;

- le diverse versioni di uno stesso spot che differivano esclusivamente per i fotogrammi di chiusura (ad esempio inviti a provare l'auto nelle concessionarie in un determinato periodo o ad approfittare delle promozioni valide in un certo arco temporale);

- gli stessi spot andati in onda su più reti televisive e dunque considerati dal sistema elementi differenti;

\footnotetext{
4 La piattaforma, raggiungibile all'indirizzo www.creativedynamix.it, offre alle aziende l'opportunità di monitorare l'attività dei concorrenti. Nielsen garantisce una elevatissima copertura mezzi e un aggiornamento pressoché costante, mettendo a disposizione dei propri clienti una serie di informazioni (quali annunci, quando, dove, con che frequenza) in grado di rappresentare un vantaggio competitivo.
} 
- gli stessi spot trasmessi in più periodi dell'anno e quindi classificati dal sistema come creatività diverse;

- le telepromozioni inserite all'interno di trasmissioni televisive;

- gli spot che si distinguevano per piccole differenze di doppiaggio;

- le versioni in lingua non italiana di uno stesso spot;

Infine, laddove presenti più versioni di differente durata di uno stesso spot (15, 20, 30, 45 o 60 secondi), si è deciso di prendere in considerazione il formato più diffuso, tanto all'interno del corpus, quanto nel sistema dei media: quello di 30 secondi. Anche in questo caso, si è trattato di una scelta in qualche modo arbitraria, ma che puntava ad aumentare il grado di uniformità dell'analisi. Alla luce di questa ulteriore selezione, il corpus si è ridotto a 490 spot di 36 case automobilistiche diverse: Alfa Romeo, Audi, Bmw, Chevrolet, Citroen, Dacia, Dr, Fiat, Ford, Honda, Hyundai, Infiniti, Jaguar, Jeep, Kia, Lancia, Land Rover, Lexus, Mahindra, Mazda, Mercedes, Mini, Mitsubishi, Nissan, Opel, Peugeot, Porsche, Renault, Seat, Skoda, Smart, Subaru, Suzuki, Toyota, Volkswagen, Volvo.

A questo punto, tenendo a mente quanto emerso dall'excursus teorico e con un corpus adeguato, definito e osservabile, si è potuto passare all'analisi, partendo dall'individuazione delle forme di valorizzazione impiegate.

\section{L'indagine empirica}

\subsection{Quattro vie maestre: pratico, utopico, critico e ludico}

Addentrandosi nell'analisi degli spot è risultato ben presto evidente che i valori di cui viene investito l'oggetto automobile sono tutti riconducibili a una delle forme individuate e illustrate originariamente da Floch. In altri termini, le caratteristiche e gli attributi per i quali viene reso desiderabile il prodotto sono vari e numerosi, ma le razionalità che sottendono e le aree semantiche che chiamano in causa sono quelle descritte dal semiologo francese. A riprova di ciò, per ciascuna forma di valorizzazione sono state individuate le occorrenze concrete emerse durante l'analisi e si è scelto di descrivere nel dettaglio uno spot particolarmente significativo. Il testo selezionato è un prototipo (cfr. Rosch e Lloyd 1978), ossia il miglior rappresentante e il caso più chiaro di appartenenza alla categoria. È stato scelto in base al possesso del maggior numero di tratti salienti rispetto alle definizioni di ciascuna forma di valorizzazione date in precedenza e ha fatto da modello sulla base del quale stabilire, per somiglianza graduale, l'appartenenza o meno di altri testi alla categoria.

VALORIZZAZIONE PRATICA

Attributi concreti presenti nel corpus: caratteristiche tecniche, optional, soluzioni funzionali, prestazioni.

Testo maggiormente rappresentativo: spot Citroen $\mathrm{C}_{3}$.

Descrizione: lo spot è incentrato sulla figura di un bizzarro individuo che guida una vecchia utilitaria, ma simula con i gesti e la voce tutta una serie di funzionalità e accessori di ultima generazione che rendono più efficace e confortevole l'utilizzo dell'auto 
Antonio Laurino | Per una nuova assiologia del consumo

(dall'antifurto ai finestrini elettrici, dall'allarme per il mancato allacciamento della cintura di sicurezza al navigatore, dall'aria condizionata ai sensori di parcheggio). A fronte di ciò, in chiusura appare l'auto pubblicizzata, naturalmente super equipaggiata.

\section{VALORIZZAZIONE UTOPICA}

Attributi concreti presenti nel corpus: spirito di avventura, libertà, elitarismo, anticonformismo, sensibilità ambientale.

Testo maggiormente rappresentativo: spot Alfa Romeo Mito.

Descrizione: nello spot si susseguono numerosi inviti che alcuni adulti, presumibilmente genitori e nonni, rivolgono a una ragazza. Si tratta di consigli a fare cose che possono essere considerate trasgressive e anticonformiste (farsi tatuare, cambiare colore dei capelli, indossare una gonna più corta, lasciare tutto e partire, acquistare una macchina "cattiva"). Al termine del video compare l'auto, e la voce off invita a "vivere rock”.

\section{VALORIZZAZIONE CRITICA}

Attributi concreti presenti nel corpus: convenienza e rapporti qualità/prezzo, prestazioni/consumi, comfort/dimensioni.

Testo maggiormente rappresentativo: spot Hyundai i2o.

Descrizione: lo spot si apre elencando l'aumento dei costi legati all'acquisto, alla gestione e alla manutenzione dell'automobile: carburanti, assicurazioni, pedaggi. Subito dopo viene presentata l'auto, di cui si sottolineano i numerosi vantaggi in termini di costi/benefici: si parte dagli optional di serie (clima, ESP, dotazioni multimediali) a fronte di un prezzo particolarmente vantaggioso, per giungere all'ottimo rapporto tra prestazioni e consumi, passando per la possibilità di usufruire di un finanziamento agevolato.

\section{VALORIZZAZIONE LUDICA}

Attributi concreti presenti nel corpus: divertimento, svago, piacere di guida. Testo maggiormente rappresentativo: spot Renault Scenic Xmod.

Descrizione: una coppia di giovani zii porta a spasso il nipote a bordo della propria auto. Annunciano al ragazzino che si dirigeranno verso alcuni luoghi di svago (il luna park, il parco, il mare), ma una volta giunti nei pressi di ciascuno di questi, non si fermano: continuano a sfrecciare con la loro auto, preferendo il piacere di guida a qualsiasi altra fonte di divertimento. Il tutto ignorando i desideri e le richieste del ragazzino.

\subsection{La quinta strada: l'estetico}

Se da un lato si registra la complessiva tenuta delle categorie flochiane, dall'altro durante l'analisi sono emersi alcuni aspetti problematici. Un primo tratto di discontinuità è legato all'importanza di cui gode la valorizzazione estetica: in un numero considerevole di casi, l'automobile viene valorizzata esclusivamente per le sue qualità estetiche $o$, di converso, per il gusto ricercato dei soggetti che la posseggono e la utilizzano. Questo dato, in sé, potrebbe apparire poco rilevante ed essenzialmente legato allo specifico settore merceologico preso in esame. Un mercato, quello dell'auto, in cui il look e la linea hanno 
una notevole rilevanza e nel quale è prassi consolidata fare ricorso a prestigiose firme del design, non solo per le vetture di fascia alta. Del resto, questo aspetto può essere significativo nella misura in cui nella formulazione originaria la dimensione estetica non rivestiva una rilevanza tale da meritare un posto all'interno dello schema flochiano. Salvo poi essere successivamente accorpata in modo piuttosto problematico a quella ludica.

Ecco perché, ai focus precedenti, dedicati alle quattro forme di valorizzazione originarie, ne va aggiunto un altro, riservato a quella estetica, che ha assunto, ormai, piena rilevanza e autonomia.

\footnotetext{
VALORIZZAZIONE ESTETICA

Attributi concreti presenti nel corpus: bellezza, fascino, aspetto esteriore, design degli interni.

Testo maggiormente rappresentativo: spot BMW Serie 6 Coupé.

Descrizione: nello spot, privo di voce narrante, si succedono dettagli di diverse parti esterne dell'auto (il muso, la fiancata, i fanali posteriori), intervallati da scorci in rapida sequenza di un'eclissi solare quasi completa. Mentre la colonna sonora incalza, lo spot culmina con una scena in cui la vettura sfreccia in una strada deserta alla luce dell'eclissi, e in sovraimpressione compare la scritta: "Uno spettacolo di rara bellezza".
}

\subsection{Sempre più forme, sempre più spesso}

Dopo aver preso atto della rilevanza assunta dalla valorizzazione estetica, si è dato uno sguardo di insieme al corpus, evidenziando come la presenza di testi all'interno dei quali si registra una copresenza di più forme di valorizzazione sia rilevante. Nella maggioranza dei casi si ricorre a valori che sono riconducibili a due dimensioni distinte, ma capita di imbattersi in spot in cui le aree semantiche sollecitate sono addirittura tre. Un caso esemplare è lo spot della Kia Carens, il quale si apre con un uomo adulto che accompagna la madre verso la sua nuova auto, in cui ci sono anche la moglie e i figli. Salita a bordo, l'anziana inizia a sottolinearne alcune caratteristiche tecniche come l'ampiezza degli interni e la grandezza del tettuccio, facendo al contempo riferimento a ciò che queste caratteristiche possono consentire da un punto di vista strettamente pratico. Dopo ogni sua affermazione, però, viene mostrato un uso ludico dell'auto, come festeggiare con tutta la squadra dopo una vittoria a calcetto, giocare a spruzzare acqua dal tettuccio aperto, oppure amoreggiare al chiaro di luna. Infine, la madre si compiace del fatto che il figlio abbia finalmente messo la testa a posto e lo spot si chiude con la frase "Diventare grandi può essere divertente", alludendo quindi a una maturità esistenziale - apparentemente - raggiunta e dotando dunque lo spot di una forte sfumatura utopica.

In generale, quasi sempre ci si trova di fronte a testi in cui le varie strategie di valorizzazione coesistono in una forma che con Claude Lévi-Strauss (1958) può essere definita mitica: si presenta il prodotto come una soluzione che riesce a tenere insieme più dimensioni che di norma vengono percepite come opposte nel senso comune. Questo può riguardare sia dimensioni 
appartenenti a forme di valorizzazione distinte - come nel caso di uno spot della Lancia Musa nel quale si assiste a una delle conciliazioni tipiche della comunicazione pubblicitaria, quella tra comodità/economicità (valori praticocritici) e lusso/raffinatezza (valori estetici) - sia valori che si situano all'interno di una sola di esse - nel caso dello spot della Kia Carens, preso in esame precedentemente, ci si muove all'interno del polo utopico, nel quale riscontriamo il farsi carico delle responsabilità di mettere su famiglia e il mantenere allo stesso tempo uno stile di vita improntato alla spensieratezza e al divertimento.

In altri casi, invece, l'opposizione non è così netta e si è piuttosto in presenza di una valorizzazione dominante alla quale vengono affiancati valori che chiamano in causa un'altra o altre due forme di valorizzazione. Se il possibile ricorso a più di una forma di valorizzazione all'interno di un singolo testo era un'eventualità prevista dallo stesso Floch, nei testi analizzati questa occorrenza appare qualitativamente rilevante, nella misura in cui una parte della distintività della proposta di un brand sembra essere affidata proprio a queste sfumature di senso, a un certo continuum di significati che, per quanto strategico, risulta poco afferrabile attraverso il modello originario. Dunque, da questo punto di vista, sembrano affiorarne i limiti ed emerge l'esigenza di elaborare una modellizzazione maggiormente soddisfacente.

\subsection{Per una nuova proposta di modellizzazione}

Riassumendo, le principali criticità del modello flochiano riguardano:

- l'irriducibilità della valorizzazione estetica a quella ludica; se da un punto di vista teorico ciò comporta l'effettiva articolazione di cinque forme di valorizzazione e non quattro sul quadrato, allo stesso tempo, nel corpus, tale valorizzazione risulta godere di notevole rilevanza e autonomia;

- l'eccessiva ampiezza semantica dei singoli termini del modello che di fatto ne intacca la tenuta logica, in particolare per ciò che riguarda la relazione di implicazione;

- la rigidità del modello del quadrato semiotico in relazione a un universo empirico fatto di testi nei quali si riscontra frequentemente la compresenza di più forme di valorizzazione, come emerso anche durante l'analisi degli spot;

- l'inadeguatezza di una modellizzazione a mapping che, a fronte della perdita di qualsiasi tipo di relazione logica tra i termini, consente di rintracciare al massimo due valorizzazioni simultaneamente.

Sulla base di queste osservazioni si propone ora una nuova modellizzazione che possa aspirare a rendere conto in modo più efficace $\mathrm{e}$ intellegibile dell'universo testuale. Essa

- non fa ricorso alla messa in quadrato, sottraendosi alle critiche mosse a questo modello;

- assegna piena rilevanza e autonomia alla valorizzazione estetica; 
Antonio Laurino | Per una nuova assiologia del consumo

- prevede che all'interno di uno stesso testo possa essere presente più di una forma di valorizzazione e consente di avanzare ipotesi in merito al diverso grado di presenza di ciascuna di esse;

- evidenzia visivamente verso quale o quali valorizzazioni tende il testo e, di conseguenza, verso quale altra propendere per un posizionamento efficace e distintivo.

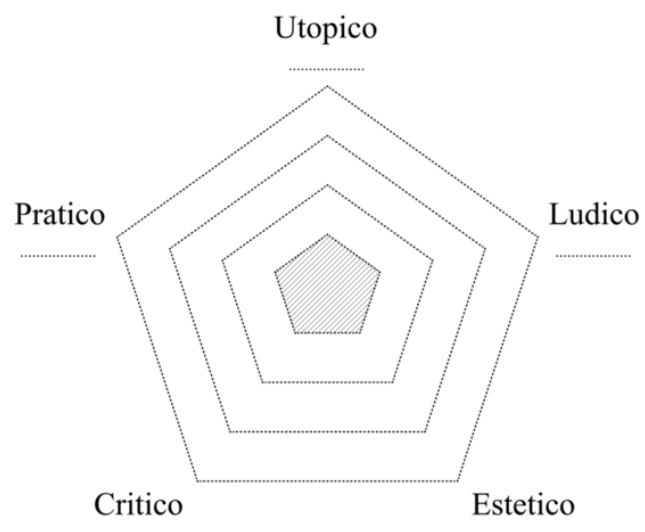

Figura 4. Nuova proposta di modellizzazione.

Come si può osservare, è stato operato innanzitutto un riposizionamento dei termini: il vertice superiore è stato riservato ai valori utopici, molto impiegati nel settore in esame e dunque investiti di una particolare rilevanza, anche visiva. Ai due lati si posizionano il pratico e il ludico, due forme di valorizzazione originariamente in relazione di contraddizione, ma che qui vengono presentate semplicemente in opposizione paritetica. Infine, nei vertici inferiori trovano posto i valori critici e quelli estetici, considerati a loro volta come opposti, dal momento che rappresentano una sorta di estremizzazione delle rispettiva logiche che sottendono la valorizzazione pratica e quella ludica: un approfondimento della razionalità pragmatica, nel primo caso, e una ancora più radicale negazione della funzionalità, nel secondo.

Inoltre, i tre pentagoni concentrici tratteggiati consentono di indicare quale o quali valorizzazioni vengono sollecitate dal testo in esame: a partire da quello centrale (pieno) è possibile fare riferimento a un diverso pentagono per indicare il diverso grado di presenza di una o più valorizzazioni. La valorizzazione principale verrà indicata tracciando una linea che, dal pentagono centrale, termina su uno dei vertici di quelli più esterni. La valorizzazione secondaria, invece, si fermerà su uno interno, a seconda della rilevanza relativa assunta nell'economia complessiva del testo.

Infine, sotto ciascuna categoria è stato previsto uno spazio dove appuntare le caratteristiche concrete rilevate durante l'analisi. Questo accorgimento evidenzia che lo schema rappresenta un modello astratto e che è indispensabile esplicitare i valori specifici messi in scena dal singolo testo. 
Antonio Laurino | Per una nuova assiologia del consumo

Per maggiore chiarezza, si proverà ad applicare il modello allo spot della Kia Carens, su cui ci si è già soffermati in 4.3. Alla luce delle forme di valorizzazione sollecitate (pratica, ludica e utopica) e sulla base del loro peso relativo all'interno del testo, lo schema assume l'aspetto seguente:

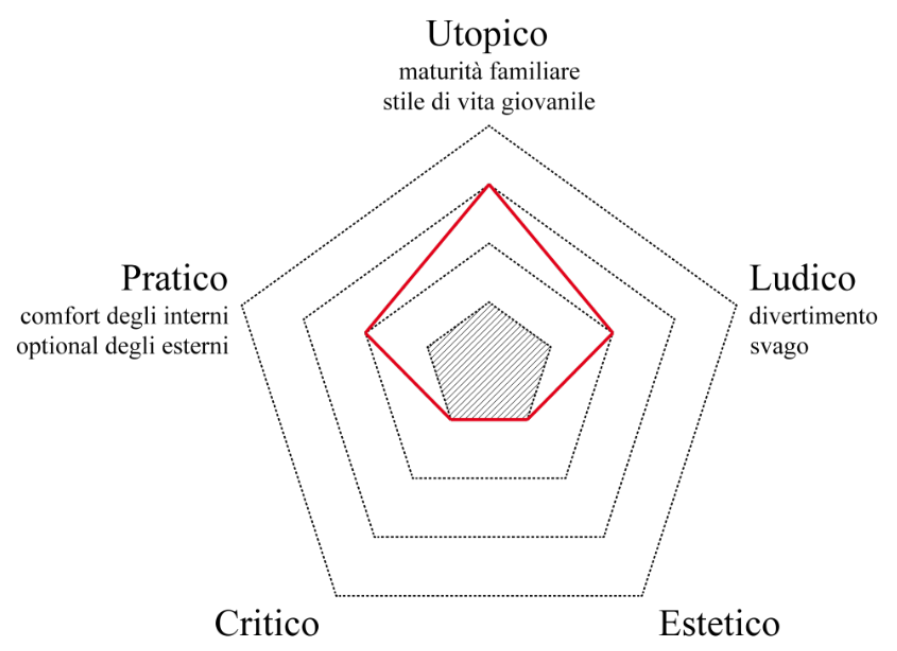

Figura 5. Applicazione del modello all'analisi dello spot della Kia Carens.

\subsection{Pit-stop: lo spazio utopico}

Dopo queste considerazioni di ordine generale, ci si è focalizzati sul polo utopico. Si è spesso sottolineato, infatti, come esso racchiuda al suo interno una costitutiva complessità. Così come teorizzato da Floch, in questa area si situano i valori esistenziali che riguardano le preoccupazioni fondamentali dell'uomo. Si tratta di un'area molto ampia, a tal punto da rischiare di rivelarsi vaga. È ormai evidente come nella comunicazione commerciale contemporanea si ricorra sempre più spesso a valori esistenziali: in determinati settori come quello in esame - il suo impiego è così frequente che individuare un tipo di valorizzazione utopica all'interno di uno spot può risultare poco significativo, nella misura in cui può non essere sufficiente per cogliere appieno il senso del testo e soprattutto per definire in cosa esso si distingue da altri che utilizzano la stessa forma di valorizzazione.

Per questo motivo, è sembrato necessario - e opportuno - andare alla ricerca di un qualche criterio di organizzazione che potesse riarticolare lo spazio semantico coperto da questo tipo di valorizzazione, inserendovi un maggior grado di intellegibilità e dunque di efficacia in fase analitica.

Osservando con attenzione gli spot che all'interno del corpus chiamano in causa valori esistenziali, si è notato che la maggior parte di essi implica un determinato modo di rapportarsi con l'Altro. Per questo motivo, la categoria semantica che è apparsa più efficace ai fini di una riorganizzazione è stata quella della /continuità (con l'Altro) Vs discontinuità (rispetto all'Altro)/. 
Con il termine "discontinuità" si indica una presa di distanza, quando non una vera e propria contrapposizione, del soggetto rispetto a ciò che lo circonda. In altre parole, qui si posizionano i valori che riguardano le tensioni e le preoccupazioni fondamentali di un individuo - o di un gruppo -, inteso come un'entità preminente rispetto alla collettività di cui fa parte. Alcuni esempi concreti ricavati dall'analisi sono il potere, la supremazia, ma anche l'indipendenza, l'autonomia, la libertà, l'anticonformismo, la trasgressione, la ribellione, l'unicità, l'elitarismo.

Il termine "continuità", invece, è utilizzato per indicare una propensione o una vicinanza del soggetto nei confronti di ciò e di chi gli sta intorno. Qui si trovano valori che chiamano in causa la vita di relazione dell'individuo e che presuppongono un rapporto contrattuale con l'Altro. Alcuni casi concreti emersi durante l'analisi sono il rispetto e la sostenibilità ambientale, l'attenzione, la cura, la sicurezza, la responsabilità per i propri cari o per il prossimo, l'altruismo.

Pur essendo solo una delle distinzioni possibili, all'interno del corpus in esame questa è apparsa la più pertinente, riuscendo ad articolare la maggior parte dei valori esistenziali investiti nell'oggetto automobile. D'altro canto, ampliando l'orizzonte di osservazione, questa categoria è apparsa ulteriormente significativa, in virtù di due aspetti che caratterizzano il discorso pubblicitario nel suo complesso: da un lato, il controverso rapporto tra pubblicità e individualismo; dall'altro, la tendenza che vede l'industria dell'advertising fare ricorso sempre più spesso a valori etici.

\subsection{1. /Discontinuità rispetto all'Altro/ e valori individualistici}

Sul rapporto tra pubblicità e individualismo si è dibattuto molto, soprattutto in ambito sociologico (cfr. Weil 1986). Edgar Morin (1995) ha osservato che la pubblicità «privilegia il consumo dei prodotti riguardanti la personalità individuale, sia nella sua sovranità individualista sia nella sua libidinalità polimorfa [...]. Inoltre, essa instilla una funzione mitologica di tipo individualista e/o libidinale in prodotti la cui funzione è diversa» (Morin 1995: 116-117).

Parallelamente, si è spesso sottolineato come la crisi delle ideologie e l'allentamento dei legami tradizionali nelle società occidentali abbiano fatto emergere un soggetto indipendente ed eclettico, un individuo la cui identità è in continua definizione e mutamento. Si tratta di un fenomeno complesso che la pubblicità ha sia cavalcato sia contribuito a generare, veicolando stili di vita e valori sociali che fanno leva sul desiderio del soggetto di distinguersi, di emergere dal continuum sociale di appartenenza, per definire la propria identità in modo oppositivo rispetto a un tutto indistinto e/o omologato. Del resto, tra i principali obiettivi della pubblicità c'è proprio il creare differenze forti e durature tra brand, prodotti e servizi, e questo, a sua volta, genera distinzioni tra i soggetti che li acquistano e li utilizzano. 


\subsection{2. /Continuità con l’Altro/ e valori etici}

L'ipotesi di centralità, all'interno della valorizzazione utopica, di valori esistenziali che sottendono una continuità con l'Altro scaturisce da tre diverse tendenze che contraddistinguono la comunicazione commerciale contemporanea.

Innanzitutto, occorre sottolineare che per rispondere alle critiche che da più parti venivano mosse al mondo dell'advertising e ai valori veicolati dalle campagne, i pubblicitari hanno iniziato a fare ricorso sempre più spesso a valori profondi, universali, coinvolgenti: amore, altruismo, solidarietà si sono diffusi sempre di più nel discorso pubblicitario. Naturalmente, dietro questa svolta "romantica" ci sono ragioni di opportunità strategica: non solo in questo modo il brand e/o il prodotto viene calato nel vissuto delle persone e dotato di un ingente capitale emozionale ma, soprattutto, fare leva su questo tipo di valori rappresenta una soluzione efficace per sfuggire a quella frammentazione degli stili di vita e delle identità che la stessa industria pubblicitaria ha contribuito a generare. Frammentazione che di fatto mette in crisi qualsiasi criterio di segmentazione: a fronte di target sempre più cangianti e smaliziati, «giocare sugli affetti elementari [...] è il metodo per raggiungere un gran numero di individui stabilendo una relazione calda» (Weil 1986: 145 tr. it.).

Accanto a questa tendenza ne va aggiunta un'altra: negli ultimi due decenni si è assistito al diffondersi di uno spiccato senso critico nei confronti del sistema del consumo e a una presa di coscienza dei suoi effetti negativi sull'ambiente naturale e socioculturale. A fronte di ciò, la pubblicità e i brand hanno messo in primo piano valori come il rispetto e la sostenibilità ambientale e hanno spinto sempre di più sulla dimensione etica dell'essere e del fare delle aziende, sia nei confronti dell'ambiente sia nei confronti dell'uomo. A ben vedere, gli esempi di prodotti e dunque di campagne che fanno leva su questi plus sono tanti e decisamente trasversali rispetto ai settori merceologici: si va dall'abbigliamento agli elettrodomestici; dai prodotti per l'igiene della casa alle campagne dei colossi dell'energia per il risparmio energetico; fino al settore automotive, nel quale la sostenibilità e il rispetto ambientale dell'oggetto automobile sono aspetti ricorrenti.

Infine, un'ulteriore tendenza è quella che vede l'universo della comunicazione pubblicitaria farsi carico sempre più spesso di valori sociali. Accade ormai frequentemente che «la marca varc[hi] i confini della comunicazione commerciale - e dei valori che tale macrogenere porta con sé - per farsi discorso sociale che invade e risucchia altri discorsi sociali (come quelli che discutono delle politiche ecologiche, della responsabilità etica nei confronti dei minori, degli aiuti ai Paesi del Terzo Mondo)» (Marrone 2007: 245). Tralasciando i problemi di legittimazione e le polemiche che tali operazioni portano sistematicamente con sé, quel che è evidente è che nella stragrande maggioranza dei casi si è di fronte, ancora una volta, a una valorizzazione utopica e più precisamente a valori esistenziali orientati verso l'Altro.

Tutto ciò - vale la pena di sottolinearlo - concentrandosi esclusivamente sulla pubblicità commerciale e lasciando dunque da parte quella sociale: un 
genere comunicativo che per sua stessa natura ricorre frequentemente a valori utopici di continuità con l'Altro, ma che, come è evidente, ha obiettivi specifici che esulano dall'universo del consumo.

Dunque, alla luce di quanto detto finora, si può affermare che l'area della /discontinuità rispetto all'Altro/ ospiti valori in qualche modo individualistici, mentre la zona della /continuità con l'Altro/ riguardi valori per lo più etici. Naturalmente, pur sostenendo la valenza pressoché generale di questa macrocategoria, è facile immaginare che non tutti i valori esistenziali di cui può essere investito un prodotto possano essere rapportati a uno di questi termini. Ad esempio, l'innovazione, valore evocato in un numero esiguo di spot, non è riconducile né all'uno né all'altro. In questo caso, si tratterebbe di un valore esistenziale neutro, ossia di un elemento che non presuppone né una continuità né una discontinuità con l'Altro.

\subsection{Trasversalità della categoria /continuità Vs discontinuità/}

Preso atto di questa prima macrocategorizzazione del polo utopico, ci si è chiesti se tale opposizione fosse rinvenibile anche nelle altre forme di valorizzazione e, soprattutto, se potesse essere utile al fine di individuare ulteriori distinzioni all'interno di ciascuna area semantica. Di fatto, passando in rassegna gli spot afferenti alle singole forme di valorizzazione, tale categoria è apparsa trasversale e dotata di una certa rilevanza. Allo stesso tempo, però, per rendere conto nella maniera più esaustiva possibile dei diversi modi in cui il rapporto con l'Altro viene declinato all'interno di ciascuna forma di valorizzazione, è stato necessario espandere la categoria semantica di partenza ricorrendo al quadrato semiotico.

Partendo dall'opposizione continuità $V s$ discontinuità sono stati generati per negazione i due subcontrari non-discontinuità e non-continuità. A questo punto, a ciascuno dei quattro termini è stato ricondotto uno specifico modo di rapportarsi con l'Altro:

- alla / continuità/ corrisponde la propensione verso l'Altro;

- alla /discontinuità/ la contrapposizione rispetto all'Altro;

- alla /non-discontinuità/ l'apertura all'Altro;

- alla /non-continuità/ la chiusura nei confronti dell'Altro.

Entrando maggiormente nel dettaglio, nell'area della propensione troviamo configurazioni narrative che si caratterizzano per un fare a favore di qualcosa o qualcuno, mentre nell'area della contrapposizione le sceneggiature prevedono un fare contro ciò che circonda il soggetto. Diverso è il caso dei due termini subcontrari: apertura e chiusura, infatti, indicano rispettivamente un fare con l'altro e un fare avulso da ciò che è attorno all'individuo. Riassumendo graficamente: 


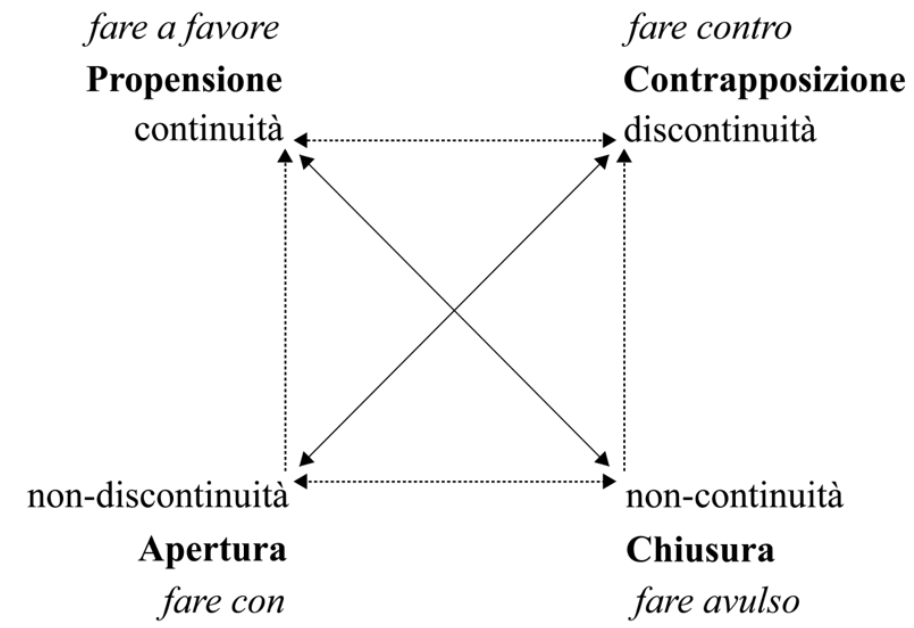

Figura 6. Quadrato semiotico dei modi di rapportarsi con l'Altro.

Naturalmente, le etichette scelte per ciascun termine sono arbitrarie, ma presentano il notevole vantaggio di essere interdefinite.

Anche se può apparire eccessivamente astratta, questa classificazione ha delle implicazioni concrete a livello analitico: facendo riferimento a essa è possibile individuare all'interno di ciascuna area semantica diverse sottoaree, corrispondenti a specifiche declinazioni testuali della categoria originaria.

I valori utopici riscontrati nel corpus si posizionano principalmente sui vertici della /continuità/ e della /discontinuità/. In altre parole, come detto, essi oscillano tra valori che sottendono una propensione verso l'Altro e valori che invece prevedono un rapporto conflittuale con ciò che sta intorno al soggetto. A ben vedere, però, anche gli altri due vertici riescono a rendere conto di ulteriori occorrenze: a quello della /non-continuità/ possono essere ricondotti gli spot in cui vi è un richiamo al proprio modo di vivere e di stare al mondo tale per cui, a fronte dello stile di vita estremamente generico evocato, l'elemento preponderante è proprio la personalizzazione, il potersi rispecchiare nell'oggetto, che diventa così custode di un mondo e protesi di un'identità esclusivamente - rispetto all'Altro - personali. Un esempio lampante di questo tipo di valori utopici si ha nello spot della Opel Adam, il cui claim è "Proprio come te"; o in quello della Ford Mustang in cui vediamo la vettura transitare lungo un'affollata via cittadina e assumere un determinato aspetto a seconda del soggetto che la guarda: uno stratagemma che rende l'auto a tutti gli effetti lo specchio della personalità di chi la possiede. Nel polo della /nondiscontinuità/, invece, troviamo alcuni spot che fanno appello a valori come la collaborazione e la condivisione, come nel caso di quello della Subaru Forester, in cui si magnifica la possibilità di fare insieme nuove esperienze e compiere piccole/grandi imprese; o di uno della Lancia Voyager, nel quale si afferma piuttosto enfaticamente che oggi "l'ego non si specchia più in sé stesso; è uno spazio che ti abbraccia per condividere ogni momento insieme a chi ami e con chi ti ama”. Inoltre, è possibile ricondurre a questo vertice anche quei 
testi in cui a essere valorizzata è la convivialità, altra declinazione della nondiscontinuità rispetto all'Altro e valore largamente evocato, ad esempio, nel settore beverage.

I valori ludici oscillano tra la /non-discontinuità/ e la /non-continuità/. Nel primo caso le sceneggiature prevedono un fare insieme a coloro e all'ambiente che circonda il soggetto: il divertimento e/o l'intrattenimento vengono intesi come un'attività da condividere con le persone che stanno intorno al soggetto. A tal proposito si possono citare diversi spot della Mini nei quali si vedono le allegre scorribande per le vie cittadine di un gruppo di amici, intesi sia come passeggeri sia come conducenti di altre auto. Nel secondo caso, invece, il soggetto si abbandona al piacere dell'utilizzo dell'oggetto e prende le distanze da ciò e da chi gli sta intorno: la fruizione ludica, dunque, avviene a discapito dei rapporti intersoggettivi. Qui si trovano numerosi spot nei quali il soggetto antepone la propria soddisfazione edonistica alle richieste e ai desideri di coloro che lo circondano, come nel caso dello spot della Renault Megane, in cui un uomo, dopo una festa, si offre di accompagnare una ragazza a casa, naturalmente con la sua nuova auto. Giunti a destinazione, però, il soggetto declina l'invito di lei a salire a casa, replicando che voleva "solo guidare".

I valori pratici si collocano sul vertice della /non-continuità/ e su quello della / continuità/. In altre parole, l'opposizione è tra soluzioni funzionali utili per i propri interessi, a prescindere da qualsiasi effetto che possono avere sull'Altro, e plus funzionali che hanno delle ricadute positive sull'essere e sul fare del prossimo. Nel primo caso si è all'interno della definizione flochiana originaria e dunque gli esempi che si potrebbero fare sono numerosissimi. Nel secondo, invece, si possono annoverare testi nei quali si fa riferimento agli elevati standard di sicurezza dell'automobile, ma a beneficiarne non è chi fruisce dell'oggetto (il conducente o i passeggeri), ma soggetti terzi, come i pedoni. Ed è proprio ciò che viene suggerito in uno spot della Volkswagen Golf, il cui protagonista è un riccio, che deve la sua vita alle caratteristiche tecniche dell'auto in termini di frenata e tenuta di strada.

I valori critici si dividono anch'essi tra /non-continuità/ e /continuità/, ossia tra soluzioni che sottendono una logica di convenienza individuale, e che non tengono conto delle esigenze altrui, e soluzioni che invece rispecchiano scelte di convenienza, per così dire, collettiva. Anche qui, nel primo caso si è di fronte al modo classico di intendere il critico e dunque gli esempi sono tanti. Nel secondo, invece, si può fare riferimento a uno spot della Honda Jazz Hybrid, giocato da un lato sulla riduzione delle emissioni e sulla convenienza che il suo utilizzo ha per l'ambiente e per il pianeta, e dall'altro sulla riduzione dei consumi con annessa convenienza per il suo possessore. In quest'ultimo caso siamo di fronte a un modo di intendere il critico che si avvicina molto a ciò che con questo termine viene indicato nel senso comune: quel consumo critico che si caratterizza per l'acquisto - e ancor prima per la valorizzazione - di beni e servizi in virtù delle loro conseguenze e implicazioni sull'intero sistema ambientale e sociale. A ben vedere, infatti, ciò che contraddistingue questo fenomeno è una razionalità volta a soppesare costi e benefici del prodotto in una prospettiva non più solamente personale, ma più ampia: gli aspetti esaminati 
Antonio Laurino | Per una nuova assiologia del consumo

vanno dalle modalità di produzione alla sostenibilità ambientale del processo produttivo; dall'eticità del trattamento accordato ai lavoratori all'eventuale attività di lobbying politica dell'azienda produttrice.

Infine, i valori estetici sembrano posizionarsi esclusivamente attorno al polo della /non-continuità/, dal momento che negli spot analizzati si riscontra una configurazione narrativa pressoché costante: il soggetto viene rapito dalla bellezza e dal fascino dell'oggetto automobile e si estranea sistematicamente dall'ambiente che lo circonda, interrompendo di conseguenza qualsiasi tipo di attività e/o relazione. Un esempio particolarmente significativo è quello di uno spot della Renault Clio, in cui si vedono numerosi soggetti raccontare l'esperienza estatica vissuta nel momento in cui hanno visto per la prima volta l'auto. Un'esperienza che li ha portati ad abbandonare ciò che stavano facendo.

In conclusione, all'interno del corpus, per ciascun tipo di valorizzazione è stato possibile rintracciare i seguenti modi di rapportarsi con l'Altro:

\begin{tabular}{|l|l|l|l|l|}
\cline { 2 - 5 } \multicolumn{1}{c|}{} & $\begin{array}{l}\text { Propensione } \\
\text { fare per } \\
\text { continuità }\end{array}$ & $\begin{array}{l}\text { Contrapposizione } \\
\text { fare contro } \\
\text { discontinuità }\end{array}$ & $\begin{array}{l}\text { Apertura } \\
\text { fare con } \\
\text { non-discontinuità }\end{array}$ & $\begin{array}{l}\text { Chiusura } \\
\text { fare avulso } \\
\text { non-continuità }\end{array}$ \\
\hline Utopico & Etica & Individualità & Condivisione & Personalizzazione \\
\hline Pratico & Benefici altrui & $/$ & $/$ & Benefici personali \\
\hline Ludico & $/$ & $/$ & Divertimento & Abbandono \\
\hline Critico & $\begin{array}{l}\text { Convenienza col- } \\
\text { lettiva }\end{array}$ & $/$ & $/$ & $\begin{array}{l}\text { Convenienza indi- } \\
\text { viduale }\end{array}$ \\
\hline Estetico & $/$ & $/$ & $/$ & Rapimento \\
\hline
\end{tabular}

Tabella 1. Forme di valorizzazione e modi di rapportarsi con l'Altro.

\section{Conclusione}

La ricognizione teorica e l'indagine empirica condotte hanno permesso di avanzare alcune ipotesi sistematiche di ampliamento, approfondimento e aggiornamento delle categorie flochiane originarie; ipotesi che, nel loro complesso, delineano una nuova assiologia del consumo. Riassumendo per punti, gli elementi di novità, rispetto allo schema elaborato da Floch, consistono:

- nell'attribuzione di maggiore importanza e piena autonomia alla valorizzazione estetica, finora problematicamente accorpata a quella ludica;

- nell'abbandono del modello del quadrato semiotico a favore di una modellizzazione a pentagono che prevede: 
- una diversa disposizione delle cinque forme di valorizzazione, non più legate da rigide relazioni di contrarietà, contraddizione e implicazione, ma al tempo stesso neppure giustapposte in modo arbitrario;

- la possibilità di rilevare la contemporanea presenza di più forme di valorizzazione all'interno di un singolo testo, permettendo, inoltre, di segnalare la rilevanza relativa di ciascuna di esse;

- nella sottoarticolazione delle cinque forme di valorizzazione in base a una categoria, quella della / continuità (con l'Altro) Vs discontinuità (rispetto all'Altro)/, che, opportunamente espansa ed espressa in termini di propensione, contrapposizione, apertura e chiusura, consente:

- di inserire un notevole grado di differenziazione laddove, in sede di analisi, risulta spesso difficile operare distinzioni tra testi riconducibili a una stessa area semantica;

- di rendere conto di tendenze e sensibilità emerse e ormai affermatesi tanto nell'universo del consumo quanto nel panorama pubblicitario dal momento dell'introduzione del modello ad oggi.

Infine, vale la pena di sottolineare che all'origine dello sforzo tassonomico profuso c'è la ferma convinzione che lo sguardo semiotico possa apportare alla comunicazione (di marketing e non solo) benefici in termini di maggiore intellegibilità, pertinenza e differenziazione (cfr. Floch 1990). Dunque, quanto qui proposto aspira, innanzitutto, a essere un insieme di strumenti analiticodescrittivi utili per fare il punto, ossia per meglio interpretare la produzione pubblicitaria contemporanea. Ma non solo. La semiotica, infatti, può trovare spazio - e utilità - pure nella fase produttivo-progettuale (cfr. Deni, Proni 2008; Bianchi, Montanari, Zingale 2010), il che vuol dire che questi modelli possono offrire anche spunti su cosa comunicare e come farlo, orientando e potenziando il processo creativo alla base dell'elaborazione di una campagna pubblicitaria. Più in concreto, ciò può avvenire: (i) partendo dall'analisi del contesto competitivo; (ii) osservando quali sono le aree semantiche maggiormente sollecitate nel particolare settore merceologico in cui si opera; (iii) scegliendo in base a queste una proposta alternativa che sia differente e rilevante per il proprio target.

\section{Bibliografia}

Bianchi, Cinzia 2005 Spot. Analisi semiotica dell'audiovisivo pubblicitario, Roma, Carocci.

Bianchi, Cinzia; Montanari, Federico; Zingale, Salvatore (a cura di) 2010 La semiotica e il progetto 2. Spazi, oggetti, interfacce, Milano, Franco Angeli.

Ceriani, Giulia

2001 Marketing moving: l'approccio semiotico. Analizzare il mix di comunicazione, gestirne gli effetti di senso, Milano, Franco Angeli.

2007 Hot spots e sfere di cristallo. Semiotica della tendenza e ricerca strategica, Milano, Franco Angeli. 
Antonio Laurino | Per una nuova assiologia del consumo

Codeluppi, Vanni

2001 Che cos'è la pubblicità, Roma, Carocci.

2005 Manuale di sociologia dei consumi, Roma, Carocci.

2010 Persuasi e felici. Come interpretare i messaggi della pubblicità, Roma, Carocci.

Codeluppi, Vanni (a cura di)

1995 La sfida della pubblicità, Milano, Franco Angeli.

Deni, Michela; Proni, Giampaolo (a cura di)

2008 La semiotica e il progetto. Design, comunicazione, marketing, Milano, Franco Angeli.

Fabris, Giampaolo

2009 Societing. Il marketing nella società postmoderna, Milano, Egea.

Ferraresi, Mauro; Schmitt, Bernd H.

2006 Marketing esperienziale. Come sviluppare l'esperienza di consumo, Milano, Franco Angeli.

Ferraro, Guido

1999 La pubblicità nell'era di internet, Roma, Meltemi.

Floch, Jean-Marie

1988 "The contribution of structural semiotics to the design of a hypermarket" in International Journal of Research in Marketing, vol. 4, n. 3. (Tr. it. "Lo spazio del mammut", in Floch 2013).

1990 Sémiotique, marketing et comunication, Paris, PUF. (Tr. it. Semiotica, marketing e comunicazione. Dietro i segni, le strategie, Milano, Franco Angeli, 1992).

1995 Identités visuelles, Paris, PUF. (Tr. it. Identità visive. Costruire l'identità a partire dai segni, Milano, Franco Angeli, 1997).

2013 Bricolage. Analizzare pubblicità, immagini e spazi, Milano, Franco Angeli.

Gensini, Stefano (a cura di)

2004 Manuale di semiotica, Roma, Carocci.

Greimas, Algirdas J.; Courtés, Joseph

1979 Sémiotique. Dictionnaire raisonné de la théorie du langage, Paris Hachette. (Tr. it. Semiotica. Dizionario ragionato della teoria del linguaggio, a cura di Fabbri P., Milano, Mondadori, 2007).

Klein, Naomi

2000 No Logo: Taking Aim at the Brand Bullies, Picador, London. (Tr. it. No logo, Milano, Baldini \& Castoldi, 2001).

Lancioni, Tarcisio

2004 "Come si legge un testo pubblicitario", in Gensini S. (a cura di), Manuale di semiotica, Roma, Carocci.

Lévi-Strauss, Claude

1958 Anthropologie structurale, Paris, Plon. (Tr. it Antropologia strutturale, Milano, il Saggiatore, 1990).

Lombardi, Marco

2007 "L'era post spot”, in Lombardi, M. (a cura di), La marca, una come noi. La personalità di marca nell'era post spot, Milano, Franco Angeli. 
Antonio Laurino | Per una nuova assiologia del consumo

Lombardi, Marco (a cura di)

1998 Il nuovo manuale di tecniche pubblicitarie. Il senso e il valore della pubblicità, Milano, Franco Angeli.

2007 La marca, una come noi. La personalità di marca nell'era post spot, Milano, Franco Angeli.

Marrone, Gianfranco

1999 C'era una volta il telefonino. Un'indagine sociosemiotica, Roma, Meltemi.

2001 Corpi sociali. Processi comunicativi e semiotica del testo, Torino, Einaudi.

2007 Il discorso di marca. Modelli semiotici per il branding, Roma-Bari, Laterza.

Marsciani, Francesco; Zinna, Alessandro

1991 Elementi di semiotica generativa, Bologna, Esculapio.

Morin, Edgar

1995 "La natura ambivalente della pubblicità" in Codeluppi, V. (a cura di), La sfida della pubblicità, Milano, Franco Angeli.

Paolucci, Claudio

2010 Strutturalismo e interpretazione, Bompiani, Milano.

Pine, Joseph B.; Gilmore, James H.

1999 The experience economy, Boston, Harvard Business School Press. (Tr. it. L'economia delle esperienze. Oltre il servizio, Milano, Etas).

Pozzato, Maria Pia

2001 Semiotica del testo. Metodi, autori, esempi, Roma, Carocci.

Rosch, Eleanor; Lloyd, Barbara B. (a cura di)

1978 Cognition and categorization, Hillsdale, Lawrence Erlbaum Associates.

Semprini, Andrea

1992 Le marketing de la marque. Approche sémiotique, Paris, Liaisons. (Tr. it. Marche e mondi possibili. Un approccio semiotico al marketing della marca, Milano, Franco Angeli, 1993).

1996 Analyser la communication. Comment analyser les images, les médias, la publicité, Paris, L’Harmattan. (Tr. it. Analizzare la comunicazione. Come analizzare la pubblicità, le immagini, i media, Milano, Franco Angeli, 1997).

2005 La marque, une puissance fragile, Paris, Vuipert. (Tr. it. La marca postmoderna. Potere e fragilità della marca nelle società contemporanee, Milano, Franco Angeli, 2006).

Testa, Annamaria

2007 La pubblicità, Roma, Carocci.

Traini, Stefano

2008 Semiotica della comunicazione pubblicitaria. Testi, marche, pratiche, consumi, Milano, Bompiani.

Weil, Pascale

1986 Et moi, emoi. La communication publicitaire face à l'individualisme, Paris, Les Editions d'Organisation. (Tr. it. Il nuovo Narciso. Comunicazione pubblicitaria e individualismo, Milano, Franco Angeli, 1990).

Volli, Ugo

2003 Semiotica della pubblicità, Roma-Bari, Laterza. 
Antonio Laurino | Per una nuova assiologia del consumo

Zannin, Andrea

2008 "Per una semiotica al lavoro. Approcci e toolkit semiotici nei progetti di marketing e comunicazione" in Deni M. e Proni G. (a cura di) 2008.

2009 Analisi, progettazione e valutazione dell'efficacia dello spot pubblicitario. Una semiotica per la ricerca di mercato, Tesi di dottorato in Semiotica, Università di Bologna.

Antonio Laurino è professore a contratto all'Università di Bologna e all'Università di San Marino. Laureato con lode in Semiotica, si occupa di scrittura professionale e didattica dell'italiano scritto. Presso l'ateneo bolognese tiene il Laboratorio di scrittura e argomentazione del corso di laurea in Scienze della comunicazione, il Laboratorio (2) di scrittura della laurea magistrale in Semiotica, il Modulo scrittura del Laboratorio di lingua italiana per studenti magistrali di area non umanistica e i laboratori di Scrittura in lingua italiana rivolti alle matricole dei diversi corsi di studio afferenti alla Scuola di Lettere e Beni Culturali; presso quello sammarinese è titolare degli insegnamenti Scrivere per il web e New business writing, nell'ambito del corso di laurea in Comunicazione e digital media. Un suo articolo, dal titolo "Amongst branding, design and architecture: a semiotic analysis of ING DIRECT's bank shops”, è apparso sull'International Journal of Marketing Semiotics \& Discourse Studies (Vol. VII, 2019). 